\title{
Theoretical Investigation of Structural, Thermodynamical and HOMO, LUMO Analysis of 6-Aminopenicillanic Acid
}

\author{
NEDA, AHMADINEJAD ${ }^{1 *}$ and MOSTAFA TALEBI TARI ${ }^{2}$ \\ ${ }^{1}$ Young Researchers and Elite Club, Arak Branch, Islamic Azad University, Arak, Iran \\ ${ }^{2}$ Young Researchers and Elite Club, Yadegar-e-Imam Khomeini (RAH), Shahr-e-Rey Branch, \\ Islamic Azad University, Tehran, Iran \\ nedaahmadinejad8810@yahoo.com
}

Received 17 October 2016 / Accepted 7 November 2016

\begin{abstract}
Quantum chemical calculations using density functional theory and 6-311G (d) basis set have been applied to analyze the interactions of $\beta$-lactam compounds and the electronic structural properties including thermochemical parameters have been investigated. The present investigation is extended to calculate the HOMO-LUMO energy gap. The calculated HOMO-LUMO energy gap shows that the charge transfer occurs within the molecule. The frontier orbital and molecular electrostatic potential surface studies have been employed to understand the active structure. The results presented in this article will be helpful to improve existing model and will open up a whole new arena of study to understand the antimicrobial characteristic from natural products against the photosensitizing DNA damage induce by 6-aminopenicillanic acid extracts and enable amuch clearer understanding of how antimicrobial drugs mediate their effects on the specific spot.
\end{abstract}

Keywords: HOMO, LUMO, DFT, 6-Aminopenicillanic acid

\section{Introduction}

The $\beta$-lactam four-membered ring (2-azetidinone system) is a unit of antibiotics of penicillin and cephalosporin types the most widely employed family of antimicrobial agents to date accounting for $50 \%$ of the word's total antibiotic market ${ }^{1,2}$. In the mid 1970s, a new class of $\beta$ lactam antibiotics characterized by a single monocyclic structure, called monobactams, were discovered $^{3}$ and some of them, of natural origin, have already been isolated and identified ${ }^{4}$. The reactive $\beta$-lactam ring is the important structural moiety for their antibacterial activity, which requires: a ring of sufficient strain; possibilities for electron delocalization outside the lactam ring and some conformational features ${ }^{5}$. Therefore, deep knowledge of the $\beta$-lactam nucleus is essential for designing a new series of these antibiotics or establishing a structureactivity relationship. The importance of 6-Aminopenicillanic acid (6-APA) as a starting point for the preparation of semisynthetic penicillins provided a strong stimulus for research into the production of this compound. Although 6-APA was first obtained as a naturally occurring 
fermentation product, the yields were always considerably lower than those of penicillin $\mathrm{G}$ or penicillin $\mathrm{V}$ obtained in fermentations in which the appropriate precursor was used. Moreover, the isolation of 6-APA from the fermentation broth was not easy. It seemed possible that an alternative route to 6-APA might be the enzymatic removal of the side-chain of the penicillin molecule. Examination of various microorganisms in our laboratories revealed that at least two types of penicillin deacylase occurred in nature ${ }^{6}$. One type was found to be widely distributed among the actinomycetes and filamentous fungi. This type of enzyme hydrolysed penicillin $\mathrm{V}$ readily but penicillin $\mathrm{G}$ was hydrolysed only slowly. The other type of deacylase was detected among bacteria; it hydrolysed penicillin $\mathrm{G}$ very readily but penicillin $\mathrm{V}$ was split only at about $20 \%$ of the rate. A process for the manufacture of 6-APA by deacylation of penicillin was first developed in our laboratories using a deacylase obtained from Streptomyces lavendulae with penicillin V as substrate; a patent for this process was filed in March 1959. Subsequently the deacylase of bacterial origin was discovered independently in a number of laboratories ${ }^{6-9}$ and a process for 6-APA production was then developed using this type of enzyme with penicillin G as substrate. With the availability of 6-APA and the opportunity to prepare semisynthetic penicillins, a number of objectives presented themselves.We have studied the theoretical investigation on the 6-APA and zwitterion of 6-APA (Figure 1). Quantum chemical analyses of 6-APA and zwitterion of 6-APA have been successful in contributing to our understanding of the frontier orbital (HOMO and LUMO) andthermochemistry of penicillin antibiotics with their target molecules.
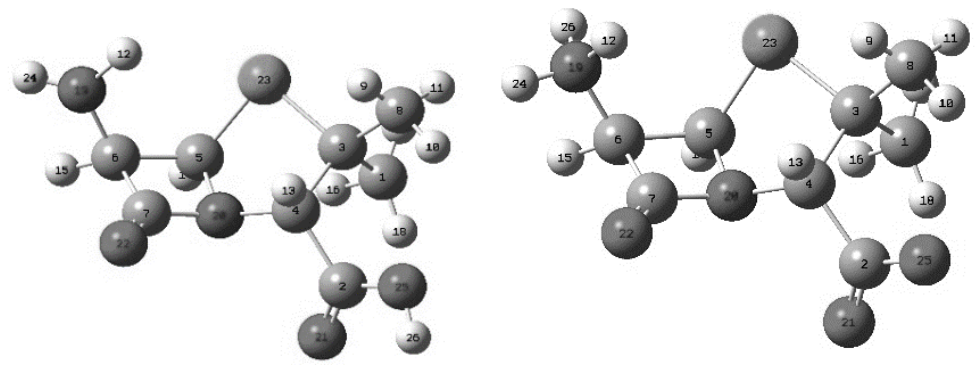

Figure 1. Molecular structure with atoms numbering of 6-APA and zwitterion of 6-APA

\section{Experimental}

All DFT calculations were performed using the Gaussian 03W program package at B3LYP method with $6-31 \mathrm{G}(\mathrm{d})$ basis set level ${ }^{10}$ with the default convergence criteria, without any constraint on the geometry ${ }^{11}$.

\section{Results and Discussion}

\section{Frontier molecular orbital analysis}

The frontier orbital (HOMO and LUMO) of the chemical species are very important in defining its reactivity ${ }^{12,13}$. Energies of HOMO and LUMO are popular quantum mechanical descriptors. It has been shown ${ }^{14}$ that these orbital's play a major role in governing many chemical reactions and are also responsible for charge transfer complexes ${ }^{15}$. The energy of the HOMO is directly related to the ionization potential and characterizes the susceptibility of the molecule towards attack of electrophiles. The energy of LUMO is directly related to the electron affinity and characterizes the susceptibility of the molecule towards attack of nucleophiles. The concept of hard and soft nucleophiles and electrophiles have been also directly related to the relative energies of the HOMO and LUMO orbital's. Hard nucleophiles 
have a low energy HOMO, soft nucleophiles have a high energy HOMO, hard electrophiles have a high energy LUMO and soft electrophiles have a low energy LUMO ${ }^{16}$. HOMOLUMO gap is an important stability index ${ }^{17}$ (Figure 2). Molecular orbital energies are molecular properties whereas orbital densities are atomic properties, and provide useful information about donor acceptor interaction ${ }^{18}$. According to frontier electron reactivity theory, the chemical reaction takes place at a position where overlap of the HOMO and LUMO are the maximum ${ }^{19}$. In the case of donor molecule the HOMO density and in case of acceptor molecule the LUMO density is important for any reaction. Frontier orbital densities can strictly be used to describe the reactivity of different atoms in the same molecule ${ }^{15-19}$. The electronegativity and hardness are of course used extensively to make predictions about chemical behavior.

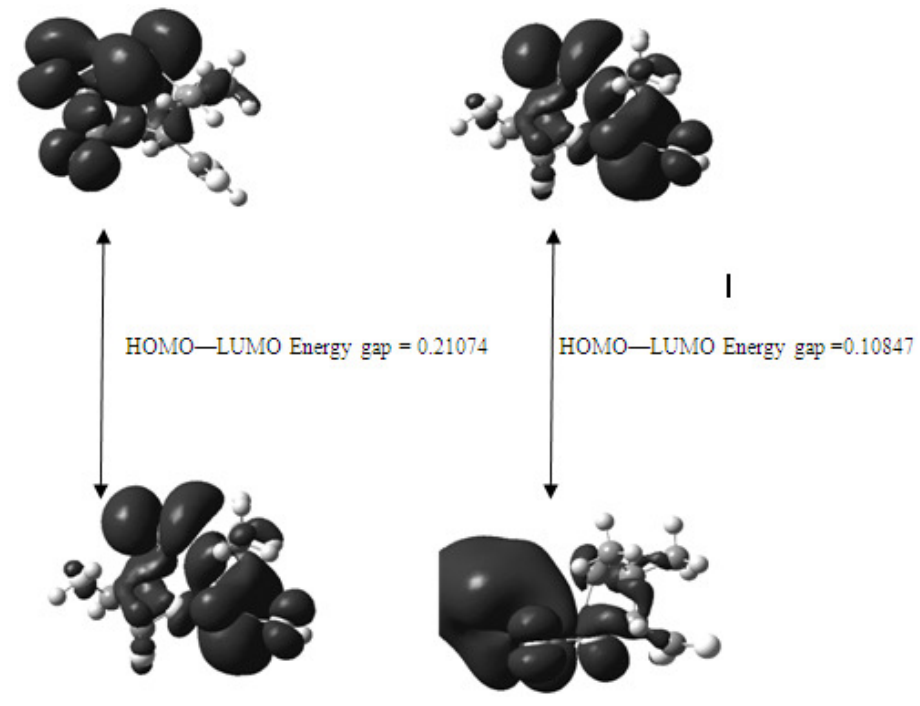

Figure 2. HOMO LUMO of Energy gap for 6-APA and witterions of 6-APA

According to, the Koopmans' theorem, Ionization potential (IE), Electron affinity (EA), Global electronegativity $(\chi)$, Chemical hardness $(\eta)$, Global softness (S) and Global Electrophilicity index $(\omega)$ can be expressed as follows.

$$
\mathrm{IE}=-\mathrm{E}_{\mathrm{HOMO}}, \mathrm{EA}=-\mathrm{E}_{\mathrm{LUMO}}, \quad X=\frac{(I E+E A)}{2}, \eta=\frac{(I E+E A)}{2}, S=\frac{1}{\eta}, \omega=\frac{\mu^{2}}{2 \eta}
$$

The highest occupied molecular orbital (HOMO) energies, the lowest unoccupied molecular orbital (LUMO) energies, hardness (g), ionization energy (IE), electronegativity $(\chi)$, total energy and dipole moment have been calculated and are given in Table 1 . The total energy of 6-APA in comparison with witterions of 6-APA is that it is more stable units; it is well-known that molecules with large HOMO-LUMO gaps are generally stable and unreactive; while those with small gaps are generally reactive ${ }^{20}$. According to our results in this table, we note that witterions of 6-APA molecule have the smallest and reactive in orbital energy difference. The ionization energy (IE) can be expressed through HOMO orbital energies as IE and electron affinity (EA) can be expressed through LUMO orbital energies $^{21}$ as EA. 
Table 1. The values of parameters of 6-APA and zwitterion of 6-APA obtained by DFT B3LYP/6-31G(d) method

\begin{tabular}{lcc}
\hline Parameters & 6-APA & Zwitterion of 6-APA \\
\hline$\in_{\text {HOMO }}(\mathrm{eV})$ & -0.24643 & -0.19584 \\
$\in_{\text {LUMO }}(\mathrm{eV})$ & -0.03569 & -0.08737 \\
Orbital energy difference & 0.21074 & 0.10847 \\
$(\Delta \mathrm{E}) \mathrm{eV}$ & & \\
Total energy (Arbitr. Units) & -1045.4247 & -1045.3504 \\
Ionization potential $(\mathrm{IE}) \mathrm{eV}$ & 0.24643 & 0.19584 \\
Electron affinity $(\mathrm{EA}) \mathrm{eV}$ & 0.03569 & 0.08737 \\
Chemical potential $(\mu) \mathrm{eV}$ & -0.14106 & -0.14160 \\
Global electronegativity $(\chi)$ & 0.14106 & 0.141605 \\
eV & & \\
Chemical hardness $(\eta) \mathrm{eV}$ & 0.10537 & 0.054235 \\
Global electrophilicity index & 0.09442 & 0.18486 \\
$(\omega)$ & & \\
Chemical softness $(\mathrm{S})$ & 9.4904 & 18.4383 \\
Dipole moment $($ Debye $)$ & 2.1057 & 16.2604 \\
\hline
\end{tabular}

The hardness $(\eta)$ corresponds to the gap between the HOMO and LUMO orbital energies. The larger the HOMO-LUMO orbital energy gap, the harder the molecule. In the present study the HOMO LUMO gap of the moleculeis $0.21074 \mathrm{eV}$ as shown in Table 1. It clearly indicates that 6-APA molecule is very stable. The ionization potential values obtained by the theoretical method also support the stability of the 6-APA molecule. The calculated dipole moment value shows that zwitterion of 6-APA is highly polar in nature.

\section{Frequency calculations}

The vibrational frequencies of 6-APA and Zwitterion of 6-APA were calculated at the DFT (B3LYP) levels of theory using the 6-31G(d) basis set ${ }^{22}$. The entropies and heat capacities were calculated using statistical mechanics based on the vibrational frequencies ${ }^{23}$. The nature of all stationary point structures were determined by analytical frequency analysis, which also provided zero-point vibrational energies (ZPEs) ${ }^{24}$. Electronic energies, enthalpies, Gibbs free energies and zero point vibrational energies for both the com- pounds using frequency calculation at $298.15 \mathrm{~K}$ temperatures are presented in Table 2 . Thermochemistry analysis follows the frequency and normal mode data.

Table 2. Thermochemistry values for 6-APA and zwitterion of 6-APAat $298.15 \mathrm{~K} 1.00$ atmosphere pressure obtained by DFT B3LYP/6-31G(d) method

\begin{tabular}{|c|c|c|c|c|c|c|c|c|}
\hline & $\mathrm{E}_{\mathrm{ZPE}}$ & $\mathrm{E}_{\text {tot }}$ & $\mathrm{H}_{\text {corr }}$ & $\mathrm{G}_{\text {corr }}$ & $\boldsymbol{\varepsilon}_{0}+\mathrm{E}_{\mathrm{ZPE}}$ & $\boldsymbol{\varepsilon}_{0}+\mathrm{E}_{\mathrm{tot}}$ & $\boldsymbol{\varepsilon}_{0}+\mathrm{H}_{\text {corr }}$ & $\boldsymbol{\varepsilon}_{0}+\mathrm{G}_{\text {corr }}$ \\
\hline 6-APA & 129.888 & 138.630 & 139.223 & 104.422 & -1045.217 & -1045.203 & -1045.202 & -1045.258 \\
\hline Zwitterion & 129.832 & 138.340 & 138.933 & 104.862 & -1045.143 & -1045.129 & -1045.128 & -1045.183 \\
\hline & $\begin{array}{c}\mathrm{E}_{\mathrm{tot}} \\
\text { (Thermal) }\end{array}$ & $(\mathrm{Cv})$ tot & $\mathrm{S}_{\mathrm{tot}}$ & $\mathrm{Q}_{\mathrm{tot}} \mathrm{BOT}$ & $\mathrm{Q}_{\mathrm{tot}} \mathrm{V}=0$ & $\begin{array}{c}\mathrm{LN}(\mathrm{Q})_{\text {tot }} \\
\text { BOT }\end{array}$ & \multicolumn{2}{|c|}{$\mathrm{LN}(\mathrm{Q})_{\mathrm{tot}} \mathrm{V}=0$} \\
\hline & 138.631 & 52.573 & 116.724 & $0.287 \mathrm{D}-76$ & $0.464 \mathrm{D}+19$ & -176.244 & \multicolumn{2}{|c|}{42.983} \\
\hline $\begin{array}{l}\text { Zwitterion } \\
\text { of 6-APA }\end{array}$ & 138.340 & 51.690 & 114.273 & $0.136 \mathrm{D}-76$ & $0.200 D+19$ & -176.987 & \multicolumn{2}{|c|}{42.144} \\
\hline
\end{tabular}

$$
E_{\text {tot }}=E_{t}+E_{r}+E_{v}+E_{e}, H_{\text {corr }}=E_{\text {tot }}+K_{B} T, G_{\text {corr }}=H_{\text {corr }}-T_{\text {stot }}, S_{\text {tot }}=S_{t}+S_{r}+S_{v}+S_{e}
$$


Sum of electronic and zero-point energies $=Æ 0+E_{\mathrm{ZPE}}$

Sum of electronic and thermal energies $=Æ 0+E_{\text {tot }}$

Sum of electronic and thermal enthalpies $=Æ 0+\mathrm{H}_{\text {corr }}$

Sum of electronic and thermal free energies $=Æ 0+\mathrm{G}_{\text {corr }}$

Where $Æ 0$ is the total electronic energy at $\mathrm{T}=0 \mathrm{~K}, \mathrm{G}_{\text {corr }}$ and $\mathrm{H}_{\text {corr }}$ represent the thermal correction to Gibbs free energy and enthalpy, respectively. The internal thermal energy $\mathrm{E}_{\text {tot }}$ is contributed from translational $\left(\mathrm{E}_{\mathrm{t}}\right)$, rotational $\left(\mathrm{E}_{\mathrm{r}}\right)$, vibrational $\left(\mathrm{E}_{\mathrm{v}}\right)$ and electronic $\left(\mathrm{E}_{\mathrm{e}}\right)$ energies, and $S_{t o t}, S_{t}, S_{r}, S_{v}$, Se are the corresponding entropies ${ }^{25}$. The next section is the individual contribution to the internal thermal energy $\left(\mathrm{E}_{\text {tot }}\right)$, constant volume molar heat capacity $\left(\mathrm{C}_{\mathrm{v}}\right)$ tot, entropy $\left(\mathrm{S}_{\text {tot }}\right)$ and partition function $(\mathrm{Q})^{26}$. The partition functions are also computed, with both the bottom of the vibrational well and the lowest (zero-point) vibrational state as reference. The comparison of thermochemistry values were calculated for two structures, which show that with increasing total energy, these values increase in both structures and also these values for 6-APA at thermochemistry are more than witterions of 6-APA which shows the greater dipole moment of 6-APA. The comparison of thermochemistry parameters of two structures show that with increasing stability the thermal correction free energy and enthalpy $\left(\mathrm{H}_{\text {corr }}\right)$, the internal thermal energy $\left(\mathrm{E}_{\text {tot }}\right)$, constant volume molar heat capacity $\left(\mathrm{C}_{\mathrm{v}}\right)_{\text {tot }}$, entropy $\left(\mathrm{S}_{\mathrm{tot}}\right)$ and the individual contributions to the internal thermal energy $\left(\mathrm{E}_{\mathrm{tot}}\right)$ increase in both structures.

\section{Conclusion}

In the present study, we used a combination of theoretical tools to compare 6-APA and witterions of 6-APA structures. The following conclusions are obtained from the current study, thermochemistry is the best tool to investigate the energy properties and andreaction mechanisms into the human organism or physiological conditions. The calculated dipole moment value shows that witterions of 6-APA is highly polar in nature but 6-APA more stable of witterions of 6-APA and 6-APA at greater internal thermal energy of witterions of 6-APA.A correlation between The larger the HOMO and LUMO orbital energy gap the stable and unreactive. Finally, the HOMO and LUMOorbital energy gap values of witterions of 6-APA is smaller than that of6-APA. In contrast, with the increase of stability values of 6-APA. So, our results reveal that the calculated HOMO and LUMO parameters of witterions of 6-APA is higher chemical softness and dipole moment.

\section{References}

1. Alcaide B and Almedros P, Chem Soc Rev., 2001, 30, 226-240;

DOI:10.1039/B007908L

2. Katzung B G, Basic and Clinical Pharmacology, Appelton \& Lange, Stamford, USA, 1998.

3. Morin R B and Gorman M, Chemistry and Biology of $b$-Lactam Antibiotics, Academic Press, New York, 1982.

4. Dürckheimer W, Blumbach J, Lattrell R and Scheunemann K H, Angew Chem Int Ed Engl., 1985, 24(4), 180-202; DOI:10.1002/anie.198501801

5. Ghuysen J M, Fre're J M, Leygh-Bouille M, Coyette J, Dusart J and Nguyen-Disteche M, Annu Rev Biochem., 1979, 48, 73-101; DOI:10.1146/annurev.bi.48.070179.000445

6. Rolinson G N, Batchelor F R, Butterworth D, Cameron-Wood J, Cole M, Eustace G C, Hart Marian V, Richards M and Chain E B, Nature, 1960, 187, 236-237;

DOI:10.1038/187236a0

7. Kaufmann W and Bauer K, Naturwissenschaften., 1960, 47(20), 474-475. 
8. Claridge C A, Gourevitch A and Lein J, Nature, 1960, 187, 237;

DOI:10.1038/187237a0

9. Huang H T, English A R, Seto T A, Shull G M and Sobin B A, J Am Chem Soc., 1960, 82(14), 3790-3791; DOI:10.1021/ja01499a083

10. Frisch M J, et al., Gaussian 03, Revision D.01 (Gaussian Inc., Wallingford CT, 2004)

11. Schlegel H B, J Comput Chem., 1982, 3(2), 214-218; DOI:10.1002/jcc.540030212

12. Fukui K, Yonezawa T and Shingu H, J Chem Phys., 1952, 20(4), 722; DOI:10.1063/1.1700523

13. Mendoza-Huizar L H and Rios-Reyes C H, J Mex Chem Soc., 2011, 55(3), 142-147.

14. Zhou Z and ParrR G, J Am Chem Soc., 1990, 112(15), 5720-5724; DOI:10.1021/ja00171a007

15. Franke R, Theoretical Drug Design Methods, Elsevier Science Ltd., London, 1984.

16. Fleming I, Molecular Orbitals and Organic Chemical Reactions, John Wiley \& Sons, London, 1976.

17. Pearson R G, Coord Chem Rev., 1990, 100, 403-425; http://dx.doi.org/10.1016/00108545(90)85016-L

18. Takahata Y and PrabhakarY S, Drug Des Delivery., 1991, 7(3), 227-239.

19. FukuiK., Theory of Orientation and Stereo Selection, vol. 39, Springer-Verlag, New York, 1975.

20. Zhang G, Musgrave C B, J Phys Chem A, 2007, 111(8), 1554-1161;

DOI:10.1021/jp061633o

21. Zhan C G, Nicholos J A and Dixon D A, J Phys Chem A, 2003, 107(20), 4184-4195; DOI:10.1021/jp0225774.

22. Jensen, James O, J Mol Struct (Theochem), 2003, 635(1-3), 211-219; DOI:10.1016/S0166-1280(03)00458-5

23. Zheng X L, Sun H Y and Law C K, J Phys Chem A, 2005, 109(40), 9044-9053; DOI:10.1021/jp058116a

24. Cherkaoui M and Boutalib A, J Mol Des., 2006, 5(8), 471-478.

25. AnW, Gao Y, Bulusu S, Zeng X C, J Chem Phys., 2005, 122(20), 204109; DOI:10.1063/1.1903946

26. Ochterski J W, Ph.D., Thermochemistry in Gaussian; June 2. 2000. 